\title{
A Window-Based Token Ring Network Supporting Two Priority Traffic Streams with Bounded Delay*
}

\author{
Mingfu Li Zsehong Tsai \\ Department of Electrical Engineering, Rm. 543 \\ National Taiwan University, Taipei, Taiwan, R.O.C. \\ E-mail: lmf@fcom.ee.ntu.edu.tw, ztsai@cc.ee.ntu.edu.tw
}

\begin{abstract}
A window-based token ring protocol supporting two priority traffic streams is proposed. This protocol uses the circulating window to limit the packet transmissions of a token holding station. Packets arrived during a window period are aluays transmitted before those arrived during subsequent window periods. Nonpreemptive priority service discipline is used to service high priority packets. $A$ call admission control policy based on delay bound is then employed to guarantee QoS such as the packet delay, delay jitter, and the packet loss-free requirement for both priority traffic streams. Under such window-based token ring operation and the call admission control, packet arrivals within a window period is guaranteed to be transmitted in the next window period and the network output traffic characteristics become predictable.
\end{abstract}

\section{Introduction}

Future high speed networks have been designed and expected to support a wide range of real-time communication services. Such real-time communication services usually need to have performance guarantees. Hence, it is important to design the medium access control policy for local area networks in order to allow packet streams of different traffic characteristics to be provided with guaranteed performance as they experienced in a high speed backbone. For high speed networks, the packet loss probability is one of the most important qualities of service. Thus, it is important to design an appropriate buffer space for the network interfaces, switches, etc. However, it is usually not easy to calculate the buffer size satisfying the packet loss probability requirement if no call admission control policy is employed. For these purposes, the concept of non-work-conserving discipline becomes popular. In [1], a new service discipline, called the Rate-Controlled Static-Priority ( RCSP) queueing discipline, is proposed to provide the throughput, delay, delay jitter, and loss-free guarantees in a connection-oriented packet-switching network. In [2], a congestion monitor strategy, called the framing strategy, for integrated services packet networks is investigated. Meanwhile, in order to provide connectivity to a large variety of users, local area networks are often connected to a high-speed backbone network such as the ATM switching network via bridge or gateway nodes, as shown in Fig. 1. For a local area network connected to a high speed backbone, the characteristics of its outgoing traffic which goes through the backbone have significant impact on the performance of the high speed backbone nodes. Therefore, it is essential to employ analytical methods to characterize the output process of a LAN IWU before reserving necessary resource on the backbone nodes. Recent works such as $[3,4]$ have focused on such issues.

*'This work was supported by National Science Council of the Republic of China under Grant NSC83-0416-E-002-12.

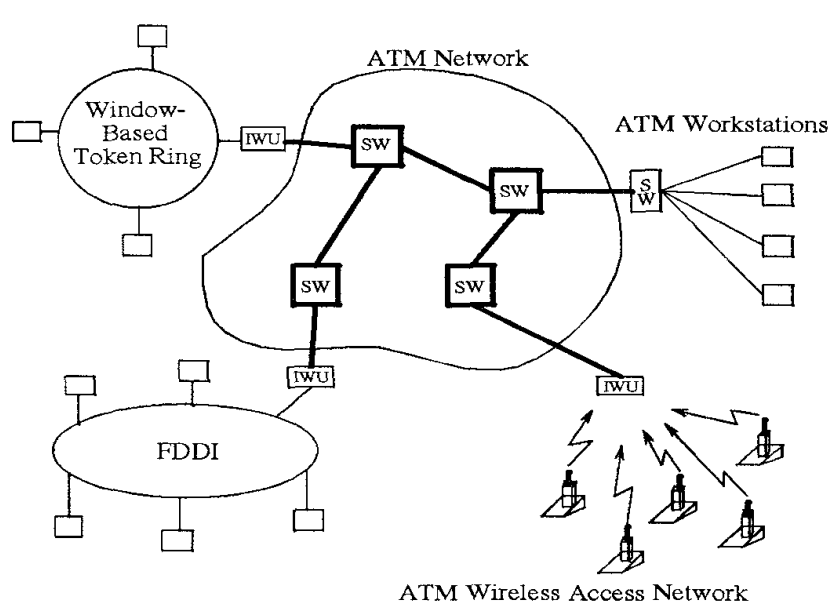

Figure 1: The internetworking environment.

Among many local area networks, the token-passing network architecture such as IEEE 802.4, 802.5, and FDDI have been popular and widely deployed for many years. In these networks, multiple priority traffic streams are supported. Recently, many new architectural designs for improving the token ring network performance on different aspects have been proposed. However, these protocols have been primarily designed with the objective of maintaining satisfactory performance on the average delay, the average throughput and the fairness $[5,6]$. In [5], token ring protocols with concurrent multiple transmissions are proposed. In [6], the helical window token ring is designed to reduce the variance of system delay and improve the fairness. In these papers, little attention is paid to the protocol design of token ring networks to the worst-case performance objectives. Therefore, facing the demand of real-time and priority traffic which has stringent constraints on the worst-case delay and jitter performance in token ring networks, we believe this is an area requiring further studies.

In this paper, we propose a new token ring protocol, called the window-based token ring protocol, for controlling possession of the token among stations. Two priority classes are supported. A call admission control policy is then employed to guarantee the packet delay, packet delay jitter and the packet loss-free requirements for both priority traffic streams.

The organization of this paper is as follows. In section 2 we describe the network model and assumptions, and then present the window-based token ring protocol. In section 3 we consider the admission control conditions that can provide performance guarantees. In section 4 , the output traffic 
characteristics are derived for both priority traffic streams. In section 5 several numerical examples are provided. Finally in section 6 we present our conclusions.

\section{The Window-Based Token Ring Protocol}

The network model and assumptions are described as follows. The token ring environment consists of $g$ stations, which can be end-stations or internetworking devices (including bridges, gateways, routers ). For each station, traffic streams of two priority classes are supported. The packet lengths of high and low priority traffic streams are constants $l_{H}$ and $l_{L}$, respectively. For the rest of this paper, we also employ the capital alphabets " $H$ " and " $L$ " to denote variables for the high and low priority traffic streams. The packet header length for both priority packets is equal to $A$. The transmission rate of the token ring is $C$. The propagation delay and bit latency from the ith station to the $(i+1)$ th station is assumed to be a constant $u_{i}$, and the ring latency for an idle token to circulate the ring one cycle is equal to $\sum_{i=1}^{g} u_{i}$, which is denoted as $u$. We also assume that the token ring network contains no packets initially.

The window-based token ring protocol is similar to the IEEE 802.5 token ring protocol. However, the window-based protocol employs the circulating window to limit the packet transmissions of a token holding station. And packets arrived during a window period are always transmitted before those arrived during subsequent window periods. Hence, the operation of the window-based token ring protocol approaches that of a single-server queueing system with the FCFS service discipline if the window size is small. This becomes the major difference between the proposed window scheme and the IEEE 802.5 token protocol. For the window-based token ring, the length of a priority- $p$ window is equal to $W_{p}$, where $p=H, L$. Here, we assume that $W_{L}$ is equal to $a W_{H}$ where $a(a>1)$ is an integer. The window boundary can be marked by the window marker which circulates the ring periodically, and we call this marking scheme as the circulation marking scheme. In order to avoid conflict between window marker and packet transmission, we assume a separate control channel is reserved on the ring. In practice, this control channel can be implemented using TDM method as in FDDI-II or directly employing a separate control link. If the clocks of all stations on the ring can be synchronized, another marking approach can be employed. In this case, each station can mark its own packets independently according to its clock based on the window lengths $W_{H}$ and $W_{L}$. This approach is called the distributed marking scheme. The advantage of this scheme is to aviod the propagation delay of the window marker and the conflict between window marker and packet transmission. In the following section, we consider the admission control conditions for both the circulation and distributed marking schemes.

In this paper, we say that the priority- $p$ packets arrived during the $n$-th priority- $p$ window period become eligible to transmit at time $T_{e}$ only when $T_{e}$ satisfies the following conditions: $T_{e}$ is larger or equal to the beginning time of the $(n+1)$-th priority- $p$ window period; all the priority- $p$ packets arrived before the $n$-th priority- $p$ window period are transmitted before time $T_{e}$. Since two priority traffic streams coexist in this token ring network, a priority mechanism similar to IEEE 802.5 reservation scheme is employed to control the access to the ring. Under this window-based token ring protocol, a station can capture the token only when it has at least one eligible packet whose priority is equal to or larger than the priority of the token. In addition, a station which has eligible high priority packets to transmit can make reservation on the busy token residing in the packet header so that high priority packets can be served as soon as possible.

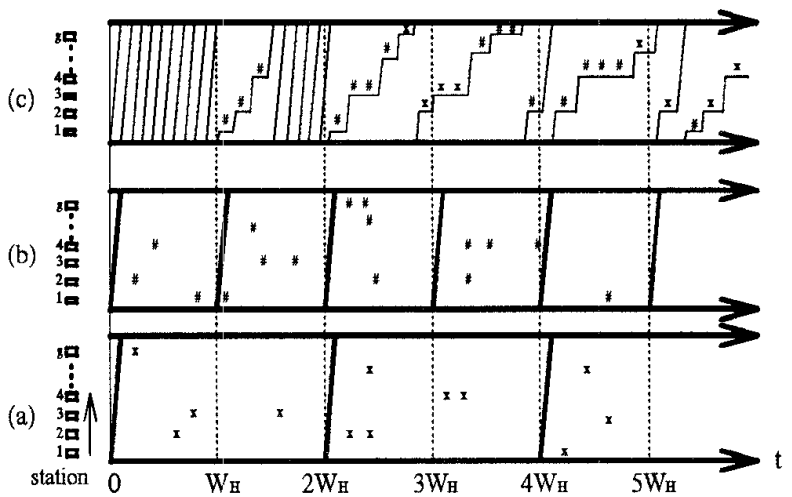

Figure 2: The window-based token ring with $W_{L}=2 W_{H}$ represented in space-tirne diagram: (a) low priority packet arrivals and low priority window marker trajectory, (b) high priority packet arrivals and high priority window marker trajectory, and (c) the token trajectory in space-time.

If no high priority packet reservation is made, then eligible low priority packets can be served immediately. After the packet header returns to the source station and no eligible packets can be transmitted, the token must be released immediately. In Fig. 2, the trajectories of the token and the window markers are shown to illustrate typical operations of the protocol, where "\#" represents the arrival of a high priority packet and " $\mathrm{x}$ " indicates the arrival of a low priority packet. Based on the above descriptions, we observe that if the token ring speed is very high ( $\geq 100 \mathrm{Mbps})$, the station may need to wait for the packet header to return when it completes a packet transmission. Thus, the minimum interval between two consecutive packet transmissions is larger than the packet service time and depends on the length of the ring latency $u$. In order to simplify the notation in the following analysis, we use $b, p=H, L$, to denote the token holding time per packet and it is given by

$$
b_{p}=\left\{\begin{array}{llll}
u+\frac{A}{C} & , & \text { if } & u>\frac{l_{p}-A}{C} \\
\frac{l_{p}}{C} & , & \text { if } & u \leq \frac{l_{p}-A}{C}
\end{array},\right.
$$

where $A$ is the length of packet header.

\section{Call Admission Control}

\subsection{Traffic Specification}

We first assume each station supports exactly one connection, which includes one high and one low priority packet streams, and an admission control mechanism is operated. Under the call admission control, each station needs to specify the traffic characteristics of its connection before the connection is admitted for both streams. We use the concept of traffic specification in [7] and the ATM Forum UNI Specification [8] and define the traffic descriptor $(X, T, \tau, l)$ for each connection. Here, $l$ is the packet length, $X$ indicates the minimum packet interarrival time, which is corresponding to the peak rate parameter. $T$ represents the minimum average packet interarrival time. Note that the inverse of $T$ is equivalent to the sustainable packet rate, or the maximum average packet rate. $\tau$ denotes the burst tolerance, which is the maximal amount of time that is allowed for the actual packet arrival to be earlier than the theoretical packet arrival time predicated, under the assumption that any two consecutive packets are separated by the interval $T$. The parameter $\tau$ is identical to that defined in the GCRA scheme 
of [8]. In other words, the traffic descriptor $(X, T, \tau, l)$ indicates the following traffic characteristics of a packet stream: each packet is of length $l$; the packet stream conforms with the GCRA scheme $(T, \tau)$; in addition, the packet interarrival time is larger or equal to $X$. The priority-p traffic stream in the ith station is specified as $\left(X_{i}^{p}, T_{i}^{p}, \tau_{i}^{p}, l_{p}\right)$, where $p=H, L$ and $i=1,2, \cdots, g$.

According to the calculation procedure in [8], the number of priority-p packet arrivals at the $i$ th station in a duration $t$ is bounded by a limit $N_{i}^{p}(t)$, which is given by ( see section 3.6.2.4.3.3 in [8] )

$$
N_{i}^{p}(t)=\min \left\{\left\lfloor 1+\frac{t+\tau_{i}^{p}}{T_{i}^{p}}\right\rfloor,\left\lfloor 1+\frac{t}{X_{i}^{p}}\right\rfloor\right\},
$$

where $|x|$ stands for the integer part of $x$. Using these traffic characteristics, the network packet delay and no loss packet transmission can be guaranteed if the call admission control operates correctly. In the following, we will show that if the admission control conditions are satisfied, the packet delay, packet delay-jitter, and packet loss-free requirements can all be met.

\subsection{Admission Control Conditions}

According to the protocol we proposed, the network performance can be guaranteed only if the packet arrival stream is strictly controlled at each station before their admission and the packet arrival pattern of each admitted stream always conforms with their traffic descriptor. In this section, we consider the admission control conditions that can guarantee the delay to be bounded under the assumptions that the priority-p traffic stream at the ith station follows the traffic specification $\left(X_{i}^{p}, T_{i}^{p}, \tau_{i}^{p}, l_{p}\right)$ for all $i$ and there are no packets at all stations initially. First, we study the condition that distributed marking scheme is employed

Theorem 1a: Assume that the distributed marking scheme is employed. According to (1), let $N_{i H}$ denote $N_{i}^{H}\left(W_{H}\right), N_{i H}^{\prime}$ denote $N_{i}^{H}\left(W_{L}\right)$, and $N_{i L}$ denote $N_{i}^{\nu}\left(W_{L}\right)$. Then the high and low priority packet delays are bounded by $2 W_{H}$ and $2 W_{L}$, respectively, if the following conditions are satisfied.

$$
\begin{aligned}
& 2 b_{L}+\sum_{i=1}^{g} N_{i H} b_{k} \leq W_{H}-u \\
& \sum_{i=1}^{g}\left(N_{i H}^{\prime} b_{H}+N_{i L} b_{L}\right) \leq W_{L}-(a+1) u .
\end{aligned}
$$

The key observation for understanding the theorem is that in each priority- $p$ window of length $W_{p}$, if the maximum total work load is no more than the time that can be used to transmitted packets, then all packets can not be delayed more than two window periods. For the circulation marking scheme, another worst case propagation delay $u$ due to the marker should be considered. Thus, we have the following theorem.

Theorem 1b: Assume that the circulation marking scheme is employed. According to (1), let $N_{i H}$ denote $N_{i}^{H}\left(W_{H}\right), N_{i H}^{\prime}$ denote $N_{i}^{H}\left(W_{L}\right), N_{i L}$ denote $N_{i}^{L}\left(W_{L}\right)$. Then the high and low priority packet delays are bounded by $2 W_{H}$ and $2 W_{L}$, respectively, if the following conditions are satisfied.

$$
\begin{aligned}
& 2 b_{L}+\sum_{i=1}^{g} N_{i H} b_{H} \leq W_{H}-2 u \\
& \sum_{i=1}^{g}\left(N_{i H}^{\prime} b_{H}+N_{i L} b_{L}\right) \leq W_{L}-(a+2) u .
\end{aligned}
$$

Using Theorems $1 a$ and $1 b$, one can then employ the following call admission policy at each station to guarantee the bounded delay for each traffic stream. When a request for a new connection arrives at the ith station, its traffic characteristics must be reported in the form of the traffic specification $\left(X_{i}^{p}, T_{i}^{p}, \tau_{i}^{p}, l_{p}\right)$. The inequalities $(2)-(3)$ or $(4)-(5)$ are then tested. If all these inequalities for both priorities are satisfied, the connection is permitted to be established. Otherwise, this request is rejected by the call admission mechanism. One can observe that the delay jitter is also bounded if the call admission control is applied. Therefore, the same call admission conditions and traffic specification method presented here can be directly applied.

\subsection{Buffer Requirement}

In order to guarantee loss-free for packets under the window-based token ring protocol, the traffic characteristics must conform to the traffic specification defined in Section 3.1 and a call admission control policy must be employed. The following theorem establishes the conditions for packet loss-free at each station, provided that the channel is error free.

Theorem 2: Assume that the call admission conditions in Theorem $1 a$ or 16 are satisfied. According to (1), if the buffer sizes $B_{i H}, B_{i L}$ for high and low priority traffic streams at the ith station are given by

$$
\begin{aligned}
B_{i H} & \geq N_{i}^{H}\left(2 W_{H}\right), \\
B_{i L} & \geq N_{i}^{H}\left(2 W_{L}\right),
\end{aligned}
$$

then there will be no packet loss at the ith station.

In Theorem 2, no assumption is made regarding the buffer sharing policy. Therefore, a total buffer of the size $B_{i H}+B_{i L}$ at the ith station is sufficient to avoid its buffer overflow.

\section{Output Traffic Characteristics}

In this section, the output traffic characteristics of a window-based token ring network with call admission control policy presented in Section 3 are derived. We employ the traffic characterization method used in [4] to determine the output traffic characteristics of the window-based token ring network with call admission control. The procedure is as follows. First, the input traffic descriptor $(X, T, \tau, l)$ is translated into Cruz's traffic characterization form (or simply called Cruz's form ) $R \sim(\sigma, \rho)$, for each input traffic stream. Next, we derive the output traffic characteristics in Cruz's form and then translate the output traffic characteristics from the Cruz's form back to the original traffic descriptor $\left(X_{\text {out }}, T_{\text {out }}, \tau_{\text {out }}, l\right)$, where the subscript out indicates the output traffic stream. Before deriving the output traffic characteristics, several notations are defined as follows.

$$
\begin{aligned}
R_{i}^{p}: & \text { packet rate function of priority- } p \text { traffic } \\
& \text { stream at station } i \\
R^{p}: & \text { aggregate packet rate function of priority- } p \\
& \text { traffic stream on the ring } \\
R_{\text {out }}^{p}: \text { output packet rate function of priority- } p & \text { traffic stream on the ring } \\
\sigma_{i}^{p}: & \text { maximum allowance of burstiness for priority- } \\
& p \text { traffic stream at station } i \\
\sigma^{p}: & \text { maximum aggregate allowance of burstiness } \\
& \text { for priority- } p \text { traffic stream on the ring, } \\
\rho_{i}^{p}: & \text { maximum average packet rate of priority- } p \\
& \text { traffic stream at station } i, \\
\rho^{p}: & \text { maximum average aggregate packet rate of } \\
& \text { priority- } p \text { traffic stream on the ring. }
\end{aligned}
$$

In the following, we will find $R_{i}^{p} \sim\left(\sigma_{i}^{p}, \rho_{i}^{p}\right)$ that satisfies the burstiness constraints. First, we know that

$$
\int_{x}^{y} R_{i}^{p} d t \leq N_{i}^{p}(y-x)
$$


and the maximum value of the long term average packet rate, $\rho_{i}^{p}$, must be equal to $\frac{1}{T_{i}^{p}}$ by the flow conservation law. Hence, from (8) and (1) we have

$$
\begin{aligned}
\int_{x}^{y} R_{i}^{p} d t & \leq 1+\frac{y-x+\tau_{i}^{p}}{T_{i}^{p}} \\
& =\sigma_{i}^{p}+\rho_{i}^{p}(y-x) \\
\sigma_{i}^{p} & =1+\frac{T_{i}^{p}}{T_{i}^{p}}, \\
\rho_{i}^{p} & =\frac{1}{T_{i}^{p}} .
\end{aligned}
$$

where

Here, we consider the sequence of time epochs at which packet transmissions terminate as the output process of the token ring. We proceed to derive the output traffic characteristics of a given input traffic with the form $R^{p} \sim\left(\sigma^{p}, \rho^{p}\right)$. According to the above definition, $R^{p}$ and $R_{i}^{p}$ are related by

$$
\int_{x}^{y} R^{p} d t=\int_{x}^{y} \sum_{i=1}^{g} R_{i}^{p} d t
$$

From (12), it is easy to observe that $\sigma^{p}=\sum_{i=1}^{g} \sigma_{i}^{p}, \rho^{p}=$ $\sum_{i=1}^{g} \rho_{i}^{p}$. Thus, we have obtained the Cruz's form for the superposition of input traffic streams. In order to derive the output traffic characteristics in Cruz's form, we need to observe the packet transmission activity within an arbitrarily given interval with non-zero length, under the proposed window-based token scheme. Let $[x, y]$ be such a nonempty interval, and let $(x-\Delta)$ be the beginning of the priority$p$ window that contains the time epoch $x,\left(y-\Delta^{\prime}\right)$ be the beginning of the priority-p window that contains $y$, where $0 \leq \Delta, \Delta^{\prime} \leq W_{p}$. Under the distributed marking scheme and the call admission control policy, the number of priority$p$ packet departures during the interval $[x, y]$ must be smaller or equal to the number of priority- $p$ packet arrivals during the interval $\left[x-\Delta-W_{p}, y-\Delta^{\prime}\right]$. Therefore, by examing the transmission activities during the interval $[x, y]$, one can derive the Cruz's form for the output process

$$
\begin{aligned}
\int_{x}^{y} R_{\text {out }}^{p} d t & \leq \int_{x-\Delta-W_{p}}^{y-\Delta^{\prime}} R^{p} d t \\
& \leq \sigma^{p}+\rho^{p}\left(y-x+\Delta-\Delta^{\prime}+W_{p}\right)
\end{aligned}
$$

Since $0 \leq \Delta, \Delta^{\prime} \leq W_{p}$, the value $\Delta-\Delta^{\prime}$ is not larger than the value $W_{p}$. By rearranging (13), one can derive

$$
\int_{x}^{y} R_{o u t}^{p} d t \leq \sigma^{p}+2 \rho^{p} W_{p}+\rho^{p}(y-x) .
$$

By (14), we directly obtain the output traffic characteristics for priority-p traffic stream in Cruz's form, as shown in the following.

$$
R_{\text {out }}^{p} \sim\left(\sigma^{p}+2 \rho^{p} W_{p}, \rho^{p}\right) .
$$

During the busy period, one can observe that the minimum packet interdeparture time $X_{\text {out }}^{p}$ is just the token holding time per packet $b_{p}$. Therefore, from equations (10) and (11), we can obtain the traffic parameters for output traffic stream$\mathrm{s}$ as follows.

$$
\begin{aligned}
T_{\text {out }}^{p} & =\frac{1}{\rho^{p}}=\frac{1}{\sum_{i=1}^{g} \frac{1}{T_{i}^{p}}}, \quad p=H, L \\
\tau_{\text {out }}^{p} & =\left(\sigma^{p}+2 \rho^{p} W_{p}-1\right) T_{\text {out }}^{p}, p=H, L
\end{aligned}
$$

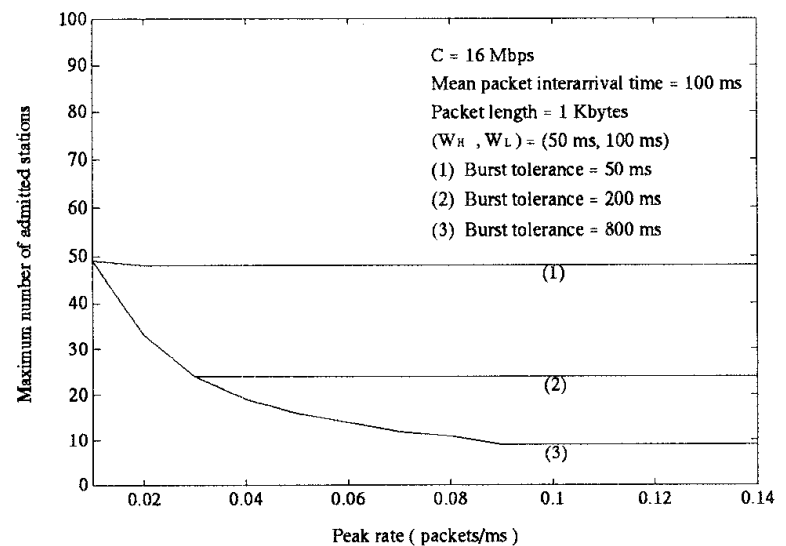

Figure 3: The maximum number of admitted stations versus the peak rate.

With these results, the output traffic descriptor can be written as $\left(b_{p}, T_{o u t}^{p}, \tau_{o u t}^{p}, l_{p}\right)$ for priority- $p$ traffic streams. When the circulation marking scheme is employed, the procedure is the same. So we omit it here.

In many occasions one may only concern the output traffic characteristics of a single connection, rather than the total traffic characteristics. In the following, we present a procedure to characterize the output traffic of a single connection while assuming that each station supports only a single connection. For the individual output traffic characteristics of the $i$-th station, its derivation is similar to that of the aggregate output traffic except that $\sigma^{p}$ and $\rho^{p}$ are replaced by $\sigma_{i}^{p}$ and $\rho_{i}^{p}$, respectively. Since the walk time between neighboring stations is arbitrary in our model, for the case that multiple connections are supported by one station, one may simply treat the end point of each connection as a "virtual" station in the model and follows the same procedure to derive the output traffic characteristics. The details are thus omitted.

\section{Numerical Examples}

In this section we consider several examples. In all examples, the cable length of the ring is $2 \mathrm{Km}$ and the zero-bit latency at each station is assumed. The packet header is equal to 10 bytes in length for all packets. We also assume that the token ring network is in symmetry. That is, the traffic specifications at all stations are the same, and at each station the traffic specification of the high priority traffic is exactly the same as that of the low priority traffic. Thus, in this section we only need to specify the parameters in $(X, T, \tau, l)$ which is the traffic specification for both priority traffic at all stations. For an active station to be admitted by the call admission control mechanism, its high and low priority traffic streams need to satisfy the call admission conditions. In the first example, the transmission rate of the token ring is 16 Mbps. It is clear from Fig. 3 that the maximum number of admitted stations decreases as the burst tolerance increases. This result is corresponding to that the throughput is decreased when the burst tolerance of the traffic stream is increased. Furthermore, one can observe that the maximum number of admitted stations is a non-increasing function of the peak rate and gradually reaches its lower bound when the peak rate exceeds a certain value. It implies that the token ring network can at least guarantee certain level of network performance regardless of the peak rate when the 


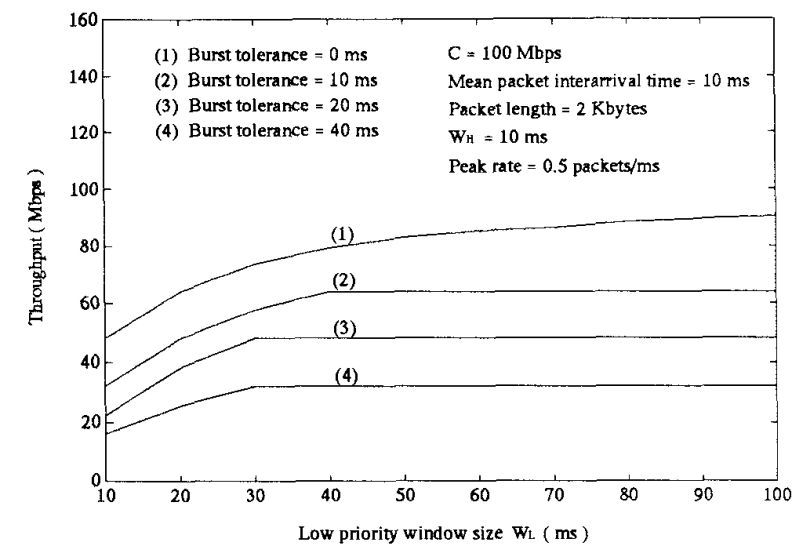

Figure 4: The maximal aggregate throughput versus the low priority window size with different burst tolerance.

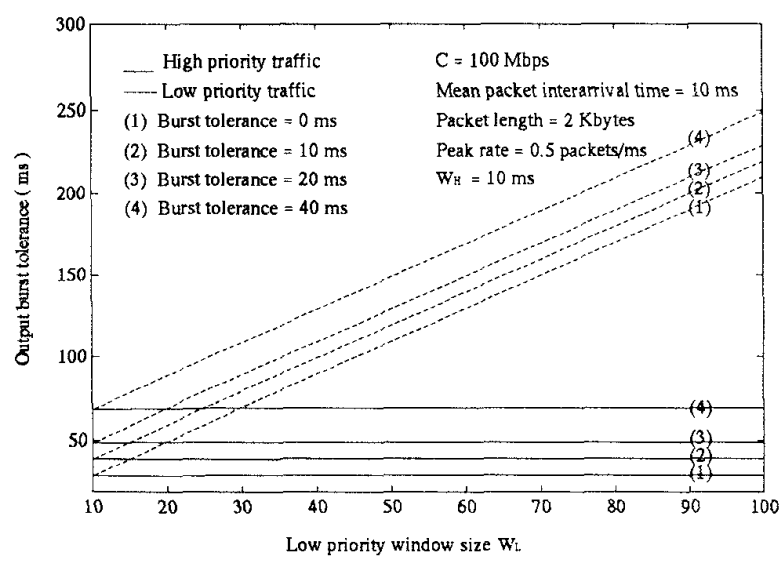

Figure 5: The upper bound of the output burst tolerance versus the low priority window size under different burst tolerance.

call admission control policy is employed.

The second example we consider is similar to the first one. But the transmission rate of the token ring is now 100 Mbps. Under the assumption that the number of admitted stations on the ring is set to be its maximal value allowed under the delay-based call admission control, the curves of the maximal aggregate throughput versus the low priority window size with various burst tolerance values are shown in Fig. 4. It reveals that the maximal aggregate throughput decreases when the burst tolerance increases and is an increasing function of the low priority window size $W_{L}$. When the burst tolerance is zero, the throughput can approach the network bandwidth ( $100 \mathrm{Mbps}$ ) if large window sizes $W_{H}$ and $W_{L}$ are chosen. For the case that the burst tolerance is of nonzero value, the maximal throughput will reach its upper bound when the low priority window size exceeds a certain threshold. In addition, the latter throughput upper bound is strictly less than the transmission rate of the ring. From Fig. 4 we also observe that in order to have high throughput, the window size must be chosen large. However, since larger window sizes yield longer packet delay, the choice of the window sizes, $W_{H}$ and $W_{L}$, is involved in a tradeoff between the packet delay/delay jitter and the network throughput.

In section 4 we have considered the output traffic characteristics as important network performance measures and have shown that it is predictable when the window-based token ring protocol is employed. Here, we present an example to illustrate such results. The network environment is the same as that in Fig. 4. The upper bound of the output burst tolerance as a function of the low priority window size is plotted in Fig. 5. Similarly, in this figure we have assumed maximal number of admitted stations. In Fig. 5, for high priority output traffic, its burst tolerance is an increasing function of the input burst tolerance and almost not varies with the low priority window size. But for low priority output traffic, its burst tolerance increases as the low priority window size $W_{L}$ or the input burst tolerance increases.

\section{Conclusions}

In this paper, a novel token ring protocol, called the window-based token ring protocol, is proposed. We then present a call admission control policy for the window-based token ring network to guarantee the packet delay, packet delay jitter, and packet loss-free requirements. Furthermore, the output traffic specifications of high and low priority traffic are predictable.

From the provided numerical examples, we observe that the network performance can be degraded seriously by the burst tolerance and/or the peak rate of the input traffic. Although the congestion and packet losses can be avoided by employing the call admission control policy, the bandwidth efficiency and the throughput can still be seriously affected. In order to improve such limitation, the statistical performance guarantee approach may be employed. We believe this is an important area that requires further studies in the future.

\section{References}

[1] H. Zhang and D. Ferrari, "Rate-Controlled StaticPriority Queueing, " IEEE INFOCOM'93, pp. 227-236.

[2] S. J. Golestani, "A Framing Strategy for Congestion Management," IEEE J. Select. Areas Commun., Vol. 9, No. 7, pp. 1064-1077, Sept. 1991.

[3] I. Stavrakakis and D. Kazakos, "Performance Analysis of a Star Topology of Interconnected Networks Under 2nd-Order Markov Network Output Processes," IEEE Trans. Commun. Vol. 38, No. 10, pp. 1724-1731, Oct. 1990.

[4] R. L. Cruz, "A Calculus for Network Delay, Part I : Network Elements in Isolation," IEEE Trans. Inform. Theory, Vol. 37, No. 1, pp. 114-131, Jan. 1991.

[5] Y. Qu and L. H. Landweber, "Parallelring : A Token Ring LAN with Concurrent Multiple Transmissions and Message Destination Removal, "IEEE Trans. Commun., Vol. 40, No. 4, pp. 738-745, April 1992.

[6] F. R. Kschischang and M. L. Molle, "The Helical Window Token Ring," IEEE Trans. Inform. Theory, Vol. 35, No. 3, pp. 626-636, May 1989.

[7] D. Ferrari and D. C. Verma, "A Scheme for Real-Time Channel Establishment in Wide-Area Networks, "IEEE J. Select. Areas Commun., Vol. 8, No. 3, pp. 368-379, April 1990.

[8] The ATM Forum, ATM User-Network Interface Specification, Version 3.0, PTR Prentice-Hall, 1993. 